UCRL-JC-123110

PREPRINT

\title{
Remote chemical sensing by laser optical pumping
}

\author{
Charles G. Stevens and Frank Magnotta
}

This paper was prepared for submittal to the

SPIE Conference

Denver, Colorado

August 5-9, 1996

August 1, 1996

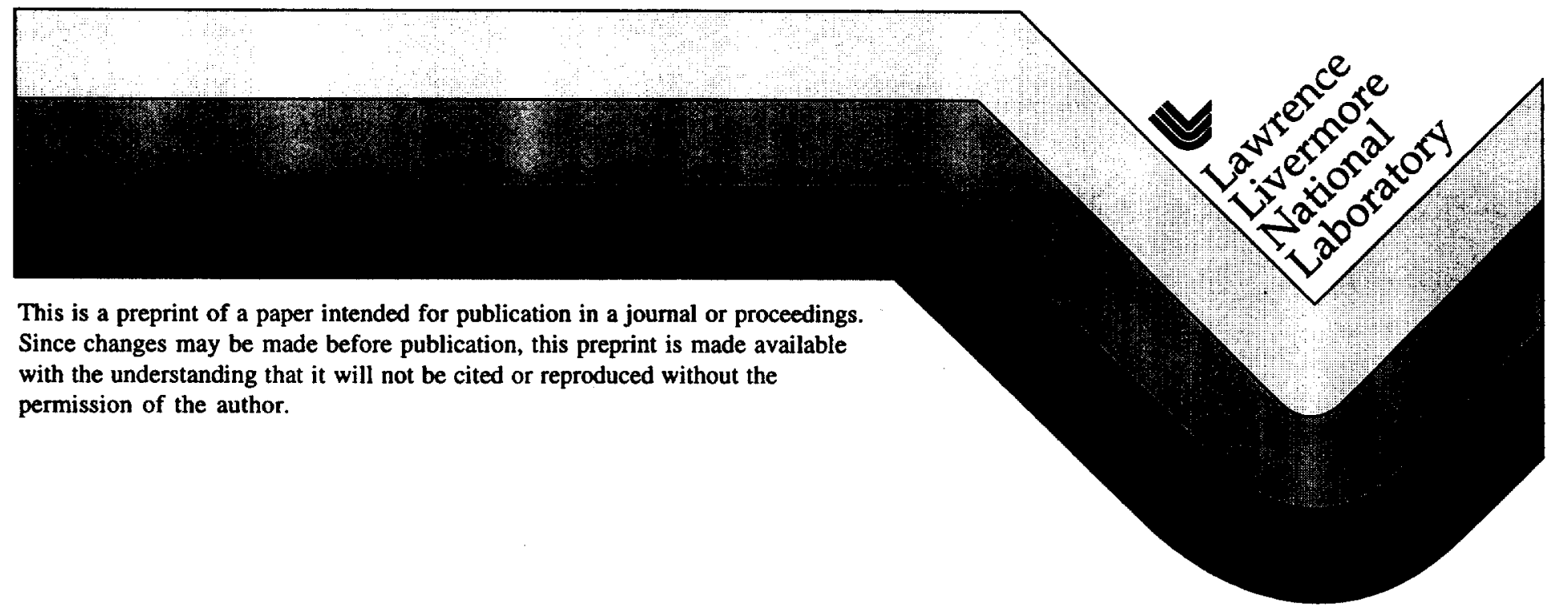




\section{DISCLAIMER}

This document was prepared as an account of work sponsored by an agency of the United States Government. Neither the United States Government nor the University of California nor any of their employees, make any warranty, express or implied, or assume any liability or responsibility for the accuracy, completeness, or usefulness of any information, apparatus, product, or process disclosed, or represents that its specific commercial products, process, or service by trade name, trademark manufacturer, or otherwise, does not necessarily constitute or imply its endorsement recommendation, or favoring by the United States Government or the University of California. The views and opinions of authors expressed herein do not necessarily state or reflect those of the United States Government or the University of California, and shall not be used for advertising or product endorsement purposes. 


\title{
Remote chemical sensing by laser optical pumping
}

\author{
Charles G. Stevens and Frank Magnotta \\ Lawrence Livermore National Laboratory \\ P.O. Box 808, Livermore, CA 94551 \\ e-mail: Stevens2@llnl.gov
}

\begin{abstract}
We are exploring a new approach to remote chemical identification that promises higher precision than can be achieved by conventional DIAL approaches. This technique also addresses and potentially solves the problem of detecting a target gas in the presence of an interfering gas or gases. This new approach utilizes an eye-safe infrared optical pumping pulse to deplete the population of a specific rotational level(s) and then sends probe pulses at the same or different wavelengths to interrogate the bleaching of the absorption. We have experimentally measured optical saturation fluence level at atmospheric pressure for $\mathrm{HCl}$, and find this level to be $\sim 1 \mathrm{~mJ} / \mathrm{cm}^{2}$, significantly below eye-safe limits in agreement with calculations. Calculations have been performed on other molecules of interest with similar results. In the laboratory, using time-delay-replicated pulses at a single frequency we have made absorption measurements with precision levels routinely approaching $0.1 \%$ after averaging 200 laser pulses. These results as well as those of two other pulse experiments will be presented.
\end{abstract}

\section{INTRODUCTION}

Conventional laser remote-chemical-sensing approaches such as DIAL (differential absorption lidar)(1) face two major difficulties in attempting to obtain high measurement accuracy and precision. One major difficulty is caused by the rapid decorrelation of speckle patterns $(2)$ with change in frequency and the consequent loss in the integrity of on-off resonance comparisons for DIAL type measurements. This can have substantial impact on the accuracy (and precision) of a measurement and requires extensive averaging over a number of independent speckle patterns. This problem is particularly serious when topological backscattering is used to enhance return signals. A second problem is caused by spectral interferences from other absorbers such as other gases, aerosols ${ }^{(3)}$ or the diffusely reflecting background. A large number of "off-resonance" wavelengths may be required to normalize the absorption spectrum and insure a proper measurement. Even with a full spectral scan there remain uncertainties in the measurement if unmodeled absorbances are present in the measurement.

This work introduces an entirely new approach to optical remote sensing that offers significant potential for reducing and/or eliminating the above difficulties. In the following paragraphs are outlined the key features of this approach followed by 
an analysis of the efficacy of the approach for a number of molecules. We show by a relatively straightforward analysis that this new approach has substantial merit and should not be difficult to implement. Following the theoretical groundwork and calculations, we then present the experimental procedure and results.

\section{RATIONALE}

The approach utilizes an optical pumping pulse to deplete the population of a specific rotational level (or set of levels) and then sends probe pulses at the same or different wavelengths to interrogate the bleaching. The approach has been provisionally named OPAL (Optical Pumping Absorption Lidar). Diagrams illustrating the several approaches to be discussed are shown in Fig. 1.

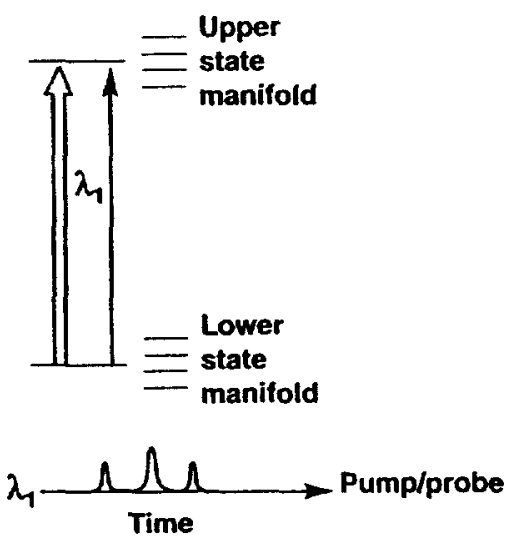

(a)
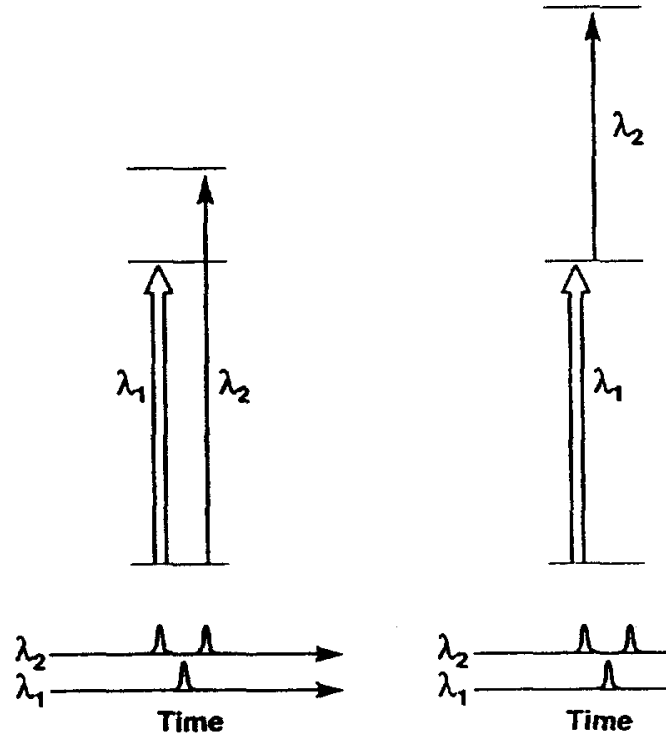

(b)

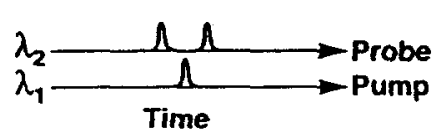

(c)

Figure 1. (a) Optical pumping pulse and probe pulses are at same frequency

The probe and pump pulses could be constructed from a single pulse in this case

(b) Probe pulses interrogate ground state depleted by pump pulse via a different transition

(c) Probe pulse monitors population accumulation in upper state by excited state absorption

Its simplest form is shown in Fig. 1(a) where two probe pulses are sent out along with the bleaching pulse, one just before the bleaching pulse and one just after it, all pulses being at the same wavelength. The intensity of Probe 1 will be attenuated by the normal molecular absorption of the species of interest. Probe 2, however, passes through the plume after the bleaching pulse and consequently suffers less attenuation. The normalized ratio of Probe 2 to Probe 1 will be a measure of the molecular absorbance at that frequency. Because the two probes are at the same wavelength, separated by a small increment in time, the problem associated with the frequency dependence of the speckle pattern disappears. This approach should provide highly precise differential absorbance measurements. The accuracy of the measurement can be separated into two parts; identification and 
quantification. Since the degree of optical pumping depends on the laser intensity at the plume, accurate molecular absorbances will depend on knowledge of this intensity. Due to atmospheric turbulence the laser beam intensity profile will not be smooth and consequently this knowledge will be uncertain by perhaps a factor of 2 .

A more difficult problem is the one of spectral interferences which seriously impair our ability to make a positive molecular identification, particularly at the lower absorbance levels ${ }^{(2)}$. This problem can be also be addressed and effectively eliminated by a variation of the bleaching approach using a different wavelength for the probe and pump pulses. This is illustrated in Fig. 1(b). Since in every molecule there are at least two optical transitions originating from a common lower rotational level (corresponding to $\mathrm{J}+1$ selection rules) a pump pulse tuned to one vibrational rotational transition, say $\mathrm{J}=+1$, an $\mathrm{R}$-branch member, will also bleach the corresponding J=-1 P-branch member. The location in frequency of the two branch members reflects characteristic vibrational and rotational energies of a molecule and in general will be a unique signature of each molecule. As a result, coincidental transitions between molecules at a pump frequency are not likely to be found at its corresponding probe frequency. Fig. 2 depicts a representation of the two color approach of Fig. 1(b), as a four frame time lapse sequence.

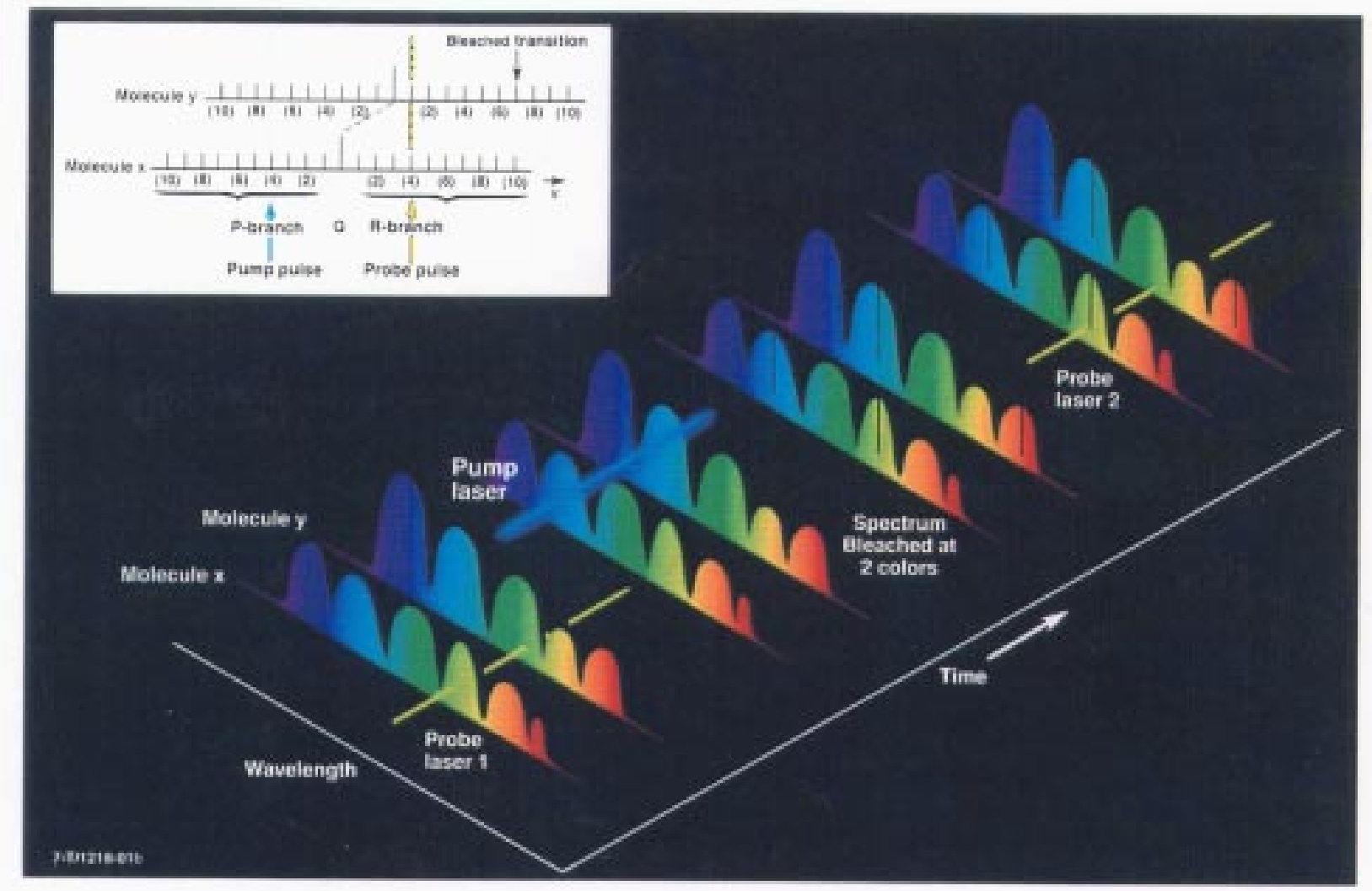

Figure 2. The two-color pump/probe technique, providing molecule specific detection is shown in four time-resolved frames. 
Molecule $\mathrm{x}$ is the target species while molecule $\mathrm{y}$ is the interferent species. In the first frame, the P-branch initial probe pulse (yellow) interrogates both $x$ and $y$ in their unperturbed state. In frame 2, the P-branch pump pulse(blue) bleaches both $x$ and $y$ molecules as shown in frame 3 . In the final frame a second probe pulse (also yellow) sees the bleached transition in molecule $x$ but not that in molecule $y$, which occurs at a different wavelength. There are other possibilities for interrogating the optical pumping. Excited vibrational state absorption, illustrated in Fig. 1(c), offers additional opportunities for selective measurements of the species of interest. This would be useful for circumventing cases where the ground state partner is obscured by atmospheric absorption. Now with regard to positive molecular identification, since there will in general be a unique relationship between pump and probe frequencies for each molecule, in principle only two wavelengths need be utilized for identification, irrespective of the shape of the absorption band. Finally, this general approach will relax the criteria for choosing absorption lines based on minimizing background absorption since the technique virtually eliminates these interferences. Conceptually then, the approach outlined above appears to offer some significant advantages over conventional DIAL approaches. The question now is whether these effects can be obtained and exploited at reasonable laser energy and power levels, recalling that the eye-safe energy fluence levels are $10 \mathrm{~mJ} / \mathrm{cm}^{2}$ in the infrared. This is addressed in the analysis that follows.

\section{GENERAL ANALYSIS OF THE APPROACH}

A general schematic of the optical pumping problem is given in Fig. 3.

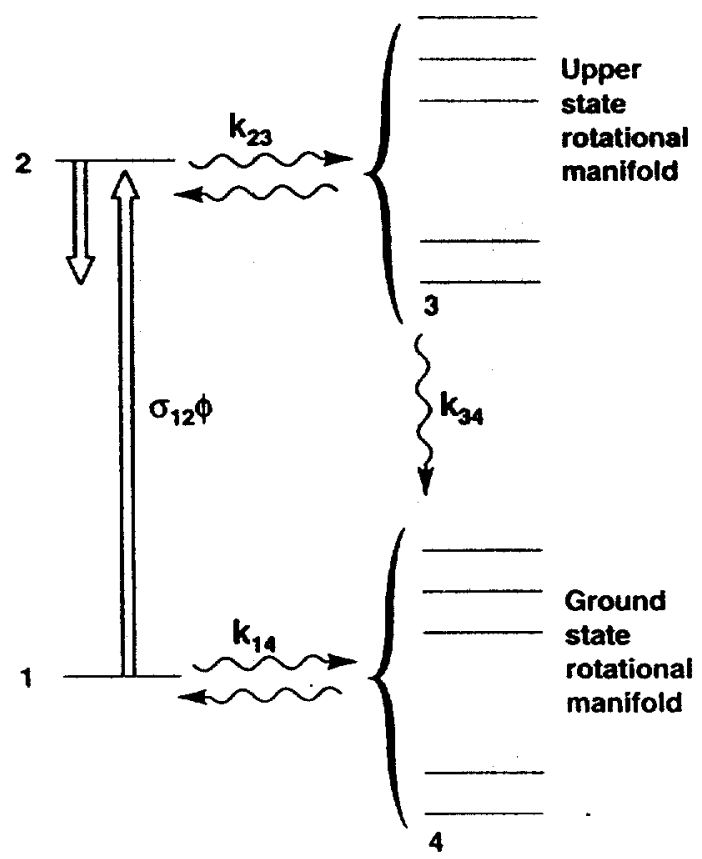

Figure 3. Kinetic relaxation routes for optical pumping analysis. $\mathbf{k}_{23}, \mathrm{k}_{14}$ refer to rotation relaxation and $\mathbf{k}_{34}$ refers to vibrational relaxation 
The problem is set by the various pumping and relaxation rates set forth there. For clarity, the population manifold is broken into two parts, separating those molecules that at $\mathrm{t}=0$ occupy the level to be pumped and the other occupied levels. The optical pumping takes place through the term $\sigma_{12} \varphi$ where $\sigma_{12}$ is the crosssection per molecule occupying a specific state and $\varphi$ is the photon flux in photons $/ \mathrm{cm}^{2} \mathrm{sec}$. The collisional relaxation term $\mathrm{k}_{23}$ is the rotational relaxation rate constant for removing population out of the initially pumped level; $k_{41}$ for the rotational infilling or Boltzmann re-distribution rate for the lower state manifold and $\mathrm{k}_{34}$ is the vibrational relaxation rate constant connecting the upper vibrational state rotational manifold to the lower state. Appropriate rate equations describing this process are straightforward.

In our analysis of small molecules with isolated transitions we will assume that the laser bandwidth is narrower than the homogeneous molecular linewidth and use peak cross-sections at line center for low signal levels. For large molecules with blended transitions, a laser bandwidth averaged cross-section is more appropriate. The optical cross-section includes the effects of power broadening by incorporating an intensity dependent term in the Lorenztian line profile. The full width at half maximum, $\gamma$, which includes the effects of short pulse widths is given by:

$$
\gamma=\frac{1}{2 \pi}\left(k_{1}+k_{2}+1 / \tau_{p}\right)+\sigma_{12} \phi
$$

where $k_{2}$ and $k_{1}$ are collisional dephasing frequencies for states 1 and $2, \sigma_{12} \phi$ the power broadening term and $\tau_{\mathrm{p}}$ is the laser pulse width. Rotational population relaxation rate constants will be somewhat smaller than dephasing frequencies, but in the absence of specific information about relaxation in the atmosphere, we will assume that relaxations out of states 1 and 2 are the same and equal to their maximum possible values, $k_{23}=\mathbf{k} 14=k r=\pi \gamma^{\circ}$, where $\boldsymbol{\gamma}^{0}$ is the small-signal-level line width.

The small-signal-level optical cross-section appropriate to the problem can be calculated from measured cross-sections by the expression

$$
\sigma_{12}^{o}=\frac{\sigma_{12(\text { meas })}^{o}}{\eta_{1}}=\frac{\sigma_{12(\text { meas })}^{o}}{\frac{g_{1}}{Q}} \exp \left[-\frac{E_{j}}{k T}\right]
$$

where $\gamma_{1}$ is the level degeneracy, Ej is the level energy, $\mathrm{kT}$ is the thermal energy and $Q$ is the partition function. The excitation cross-section is therefore greater than the measured cross-section by the ratio of the total population to the population residing in the pumped level, a large value in some cases. In order to take advantage of this larger cross-section and ensure minimum pulse energy requirements, the optical pumping must be done on a time scale that is comparable 
to the collisional relaxation time for rotational levels at atmospheric pressures. The optical cross-section, including the effects of power broadening, is then given by

$$
\sigma_{12}=\frac{\sigma_{12}^{\mathrm{o}} \gamma^{\mathrm{o}}}{\gamma}
$$

We can estimate the intensity levels necessary to achieve significant population transfer by considering the problem as a two-level system. This is a standard problem ${ }^{(4)}$ and after a bit of algebraic manipulation using a statistical density matrix formalism, an expression for population change as a function of photon flux on line center can be derived.

$$
\frac{\mathrm{n}_{1}-\mathrm{n}_{2}}{\mathrm{n}_{1}^{\circ}}=\frac{1}{1+\sigma_{12}^{\circ} \phi \mathrm{t}_{\mathrm{r}}}
$$

where $n_{1}^{\circ}$ is the ground state population prior to the laser pulse, $\sigma_{12}^{\circ}$ is the peak optical cross-section, $\phi$ is the photon flux in photons $/ \mathrm{cm}^{2}$ sec and $\tau_{\mathrm{r}}$ the relaxation time constant for the population difference between states 1 and 2 and is equal to $1 / \mathrm{k}_{\mathrm{r}}$. Using a laser pulse with a time duration equal to the relaxation time, the photon fluence (photons $/ \mathrm{cm}^{2}$ ) required to decrease the population difference to $1 / 2$ its original value is given by the reciprocal of the optical cross-section. A few representative examples of saturating fluences are given in column 7 in Table 1. As can be seen the fluence requirements are rather modest. Included also in the table is an estimate of the bleaching signal expected at saturating fluences for a plume areal concentration of $1 \mathrm{ppm}-\mathrm{m}$. The small molecule estimates are probably good to better than a factor of 2 . In the larger molecules, particularly spherical tops like $\mathrm{SF}_{6}$, acStark effects can come into play causing state splittings and mixings that diminish the pumping efficiencies. The magnitudes of these effects are best left to measurement.

\begin{tabular}{|l|c|c|c|c|c|c|c|}
\hline Molecule & $v\left(\mathrm{~cm}^{-1}\right)$ & $\begin{array}{c}\text { Transition } \\
\text { Fraction }\end{array}$ & $\sigma^{\circ}$ (meas) & Population & $\sigma^{\circ}\left(\mathrm{cm}^{2}\right)$ & $\begin{array}{c}\mathrm{J}_{\text {sat }} \text { (mjoules/cm } \\
\text { @ saturation }\end{array}$ & Signal/ppm-m \\
\hline $\mathrm{HF}$ & 4039 & $\mathrm{R}(1)$ & $8 \times 10^{-18}$ & 0.22 & $4 \times 10^{-17}$ & 1.6 & $1 \times 10^{-2}$ \\
\hline $\mathrm{HCl}$ & 2776 & $\mathrm{P}(5)$ & $2 \times 10^{-18}$ & 0.1 & $2 \times 10^{-17}$ & 2.6 & $3 \times 10^{-3}$ \\
\hline $\mathrm{NO}$ & 1900 & $\mathrm{R}(6)$ & $3 \times 10^{-19}$ & 0.019 & $1.6 \times 10^{-17}$ & 2.4 & $4 \times 10^{-4}$ \\
\hline $\mathrm{NO}_{2}$ & 2920 & $\mathrm{R}(16$ & $2.2 \times 10^{-19}$ & 0.014 & $1.6 \times 10^{-17}$ & 3.8 & $2 \times 10^{-4}$ \\
\hline
\end{tabular}

Table 1. Calculated saturation fluences and signal levels for a variety of molecules 
The above two-level analysis gives us an early time estimate of population transport in these molecules. To get a better picture of the dynamics of population transport, the rate equations for the effective four-level system are solved. A program incorporating the fourth order Runge-Kutta method for solving simultaneous differential equations was used and the HF R(l) transition examined.

The rotational relaxation constants, $k_{41}$ and $k_{23}$ are derived directly from the linewidth of the transition, again, assuming that population is transferred on every collision. This places an upper bound on the relaxation rate for the problem. The vibrational relaxation constant at one atmosphere in air is measured to be 0.037 nsec $^{-1(5)}$. This represents a population shelving for a fairly long time. The calculated results are displayed in Fig. 4. The laser pulse profile is chosen to be Gaussian in shape with a FWHM of 100 psec. In this example, the laser excitation rate $\sigma^{0}{ }_{12} \phi$ at its peak is chosen at $10 \mathrm{nsec}^{-1}$, the same as the population relaxation rate. This is the same excitation rate as the saturation rate in the 2-level system. At the same fluence found to saturate the 2-level system, the full analysis shows a population difference of 0.42 instead of 0.5 . The dependence of bleaching signal as a function of laser fluence for HF is shown in Fig. 5. Significant signal is evident even at $1 \mathrm{mj} / \mathrm{cm}^{2}$.

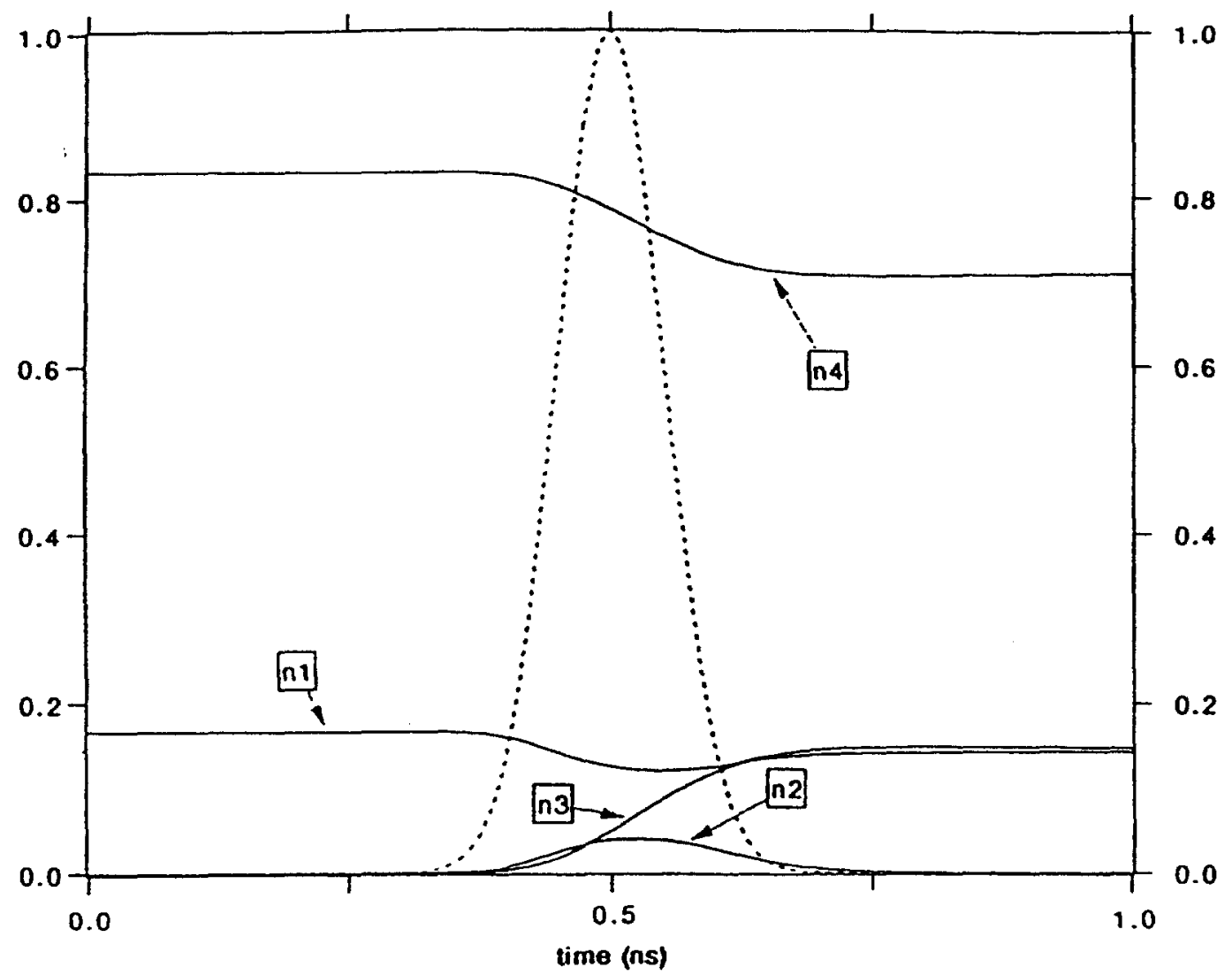

Figure 4. Population dynamics for the optical pumping of $H F$ molecule at the $R(1)$ transition at $4039 \mathrm{~cm}^{-1}$. The Gaussian laser pulse (100 psec FWHM) has been chosen to give a peak excitation rate of $1010 \mathrm{sec}^{-1}$. The long time depletion of population is due to accumulation in the upper vibrational manifold. 


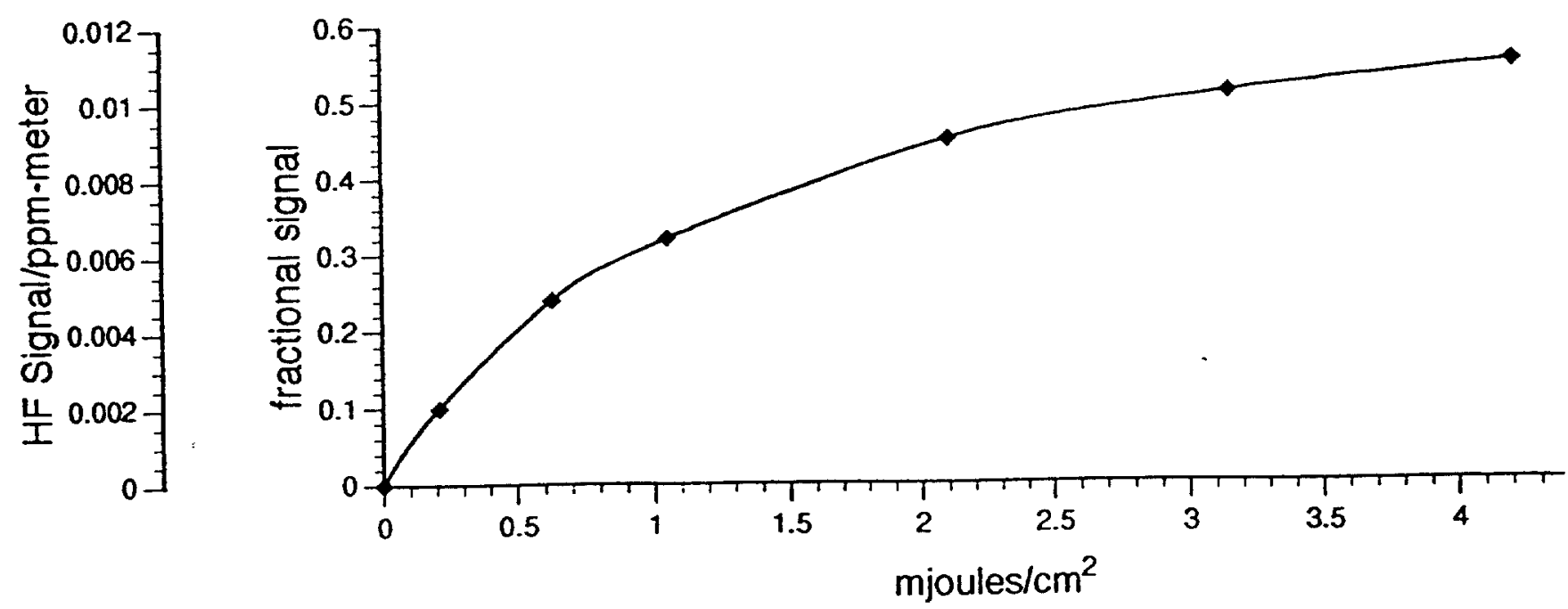

Figure 5. Saturation curve for $\mathrm{R}(1)$ transition in HF showing the degree of bleaching as a function of laser fluence. Also shown is the optical transmission enhancement at an areal concentration of $1 \mathrm{ppm}-\mathrm{m}$.

Approximately $20 \%$ of the population has been transferred from the ground state to the upper vibrational state during the pulse. This population recovers with the time constant characteristic of vibrational relaxation. In general, this time constant ranges from 10 to 1000 times smaller than rotational relaxation. We report here experiments on the saturation of the $\mathrm{R}(4)$ line of $\mathrm{HCl}$. Fig. 6 shows the results of modeling this transition using a laser pulse width of $4 \mathrm{nsec}$, equivalent to our laboratory laser. The population dynamics is modeled using an excitation rate of 1 $\mathrm{nsec}^{-1}$. This is equivalent to a $12 \mathrm{~mJ} / \mathrm{cm}^{2}$ laser pulse fluence. Significant population transfer between levels 1 and 2 is predicted. The absorption averaged over the pulse length has decreased by $32 \%$ in this example. This clearly shows the possibility of making differential absorbance measurements by ratioing a weak pre-pulse with a significantly stronger bleaching pulse. The pulse pair used in this measurement requires a single transient digitizer of modest capability. 


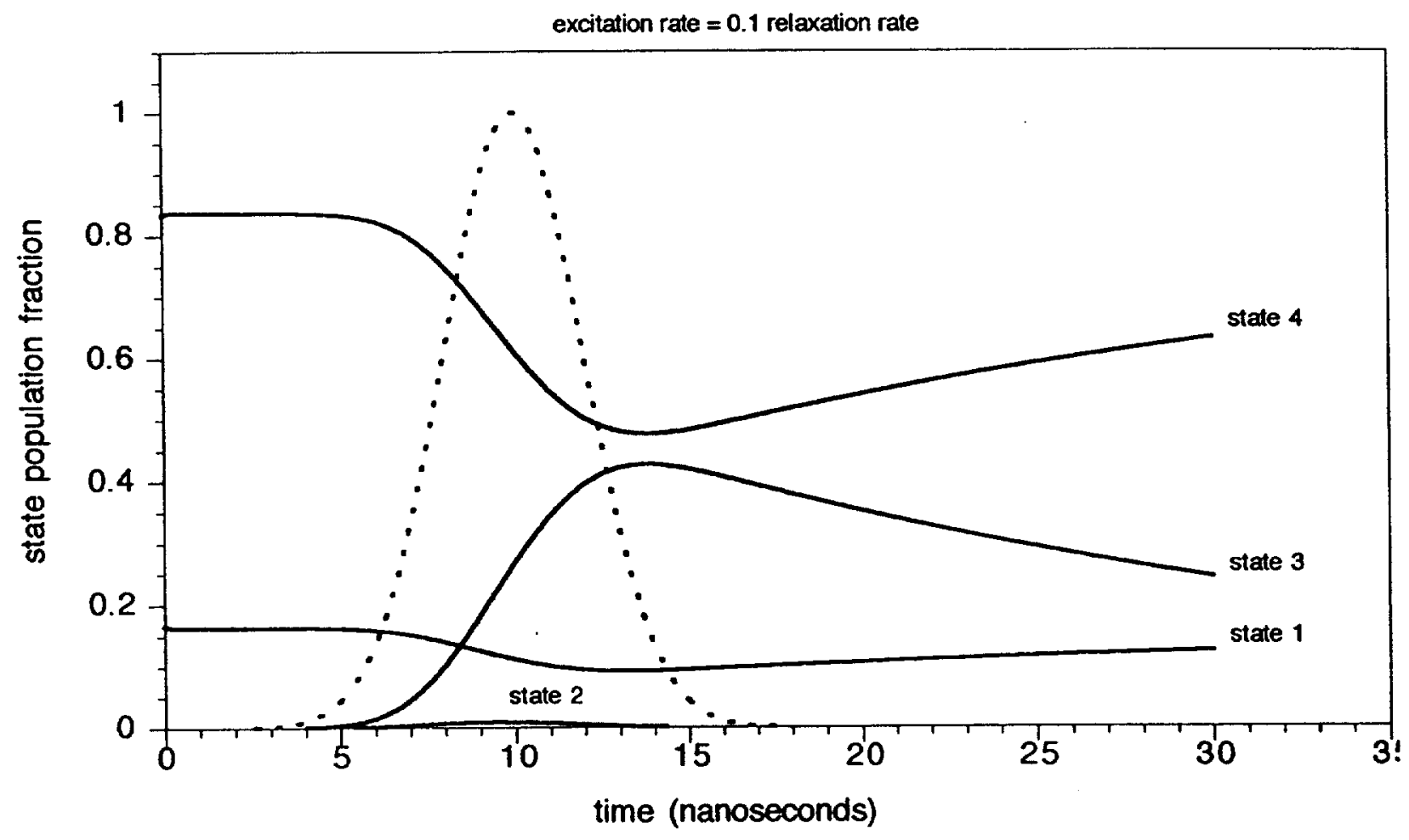

Figure 6. Population dynamics following optical pumping of the $\mathrm{R}(4) \mathrm{HCl}$ transition using a $4 \mathrm{nsec}$ Gaussian laser pulse and a peak excitation rate of $1 \mathrm{nsec}^{-1}$.

\section{EXPERIMENTAL PROCEDURE AND RESULTS}

Two separate sets of experiments were performed, the first to determine the bleaching behavior of $\mathrm{HCl}$ and the second to measure absolute measurement precision and signal response using a single detector. The first setup splits the beam spatially in two as in a conventional absorption experiment, while the second splits the beam temporally with the use of a variable delay multipass cell. Fig. 7 depicts the basic optical layout consisting of a commercial tripled Nd:YAG pumped OPO. The residual second harmonic at $532 \mathrm{~nm}$ is combined with the OPO signal output (shown as $623 \mathrm{~nm}$ ) in a $\mathrm{LiNbO}_{3}$ crystal to generate several millijoules of tunable difference frequency (shown as $3.6 \mu \mathrm{m}$ ) in the mid-IR, at a bandwidth of around 0.2 $\mathrm{cm}^{-1}$. A portion of the beam is sent through a separate reference cell to monitor the wavelength overlap with the desired absorption feature. The signal from two detectors is ratioed as a function of laser fluence and number of laser pulses. 


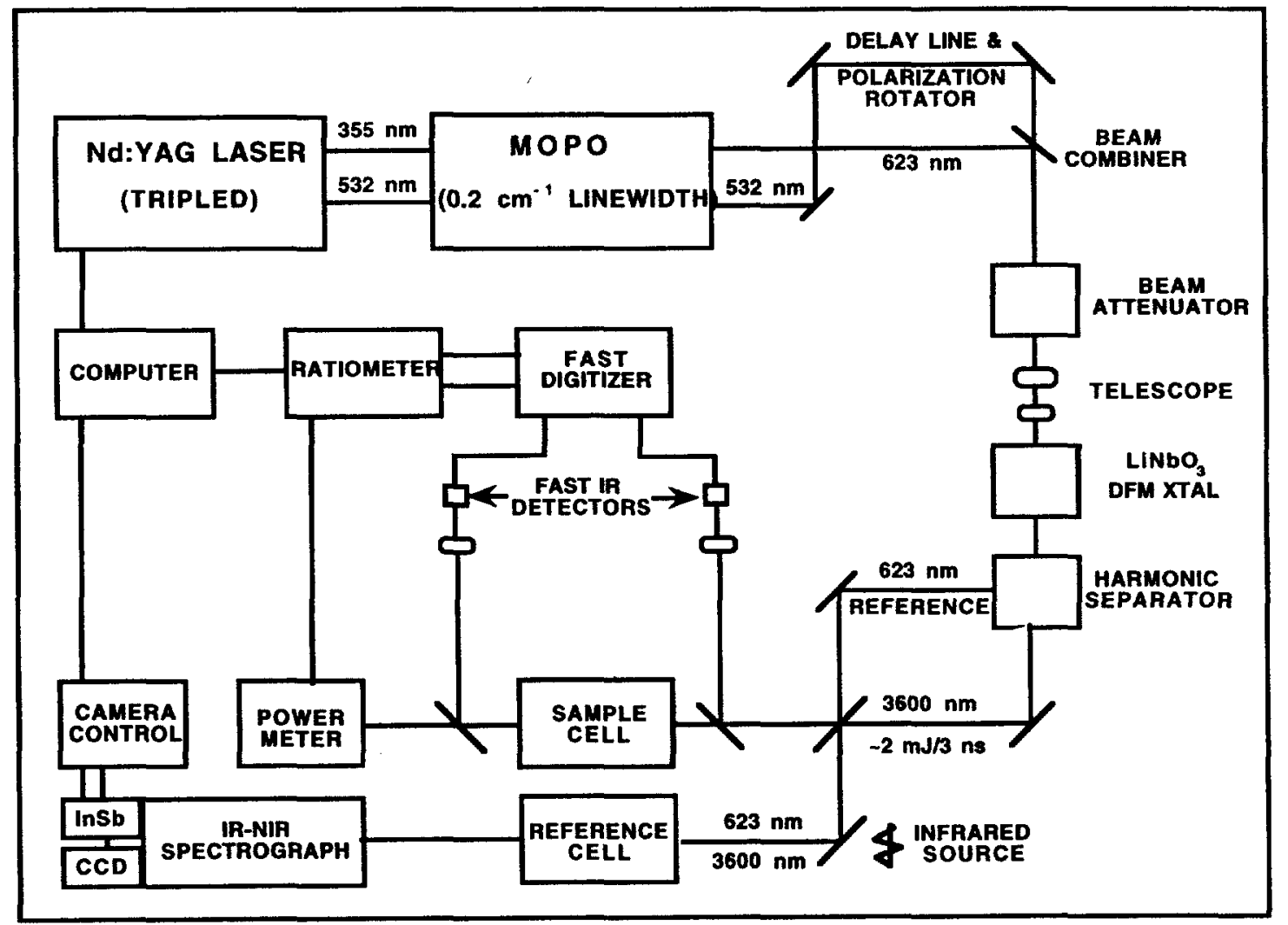

Figure 7. Basic optical layout for saturation experiments

The bleaching results for $\mathrm{HCl}$ as a function of laser fluence are shown in Fig. 8. Also shown are the results of a four-level rate equation calculation in which the rotational relaxation rate constant $\left(k_{23}\right)$ was adjusted to fit the experimental data. 


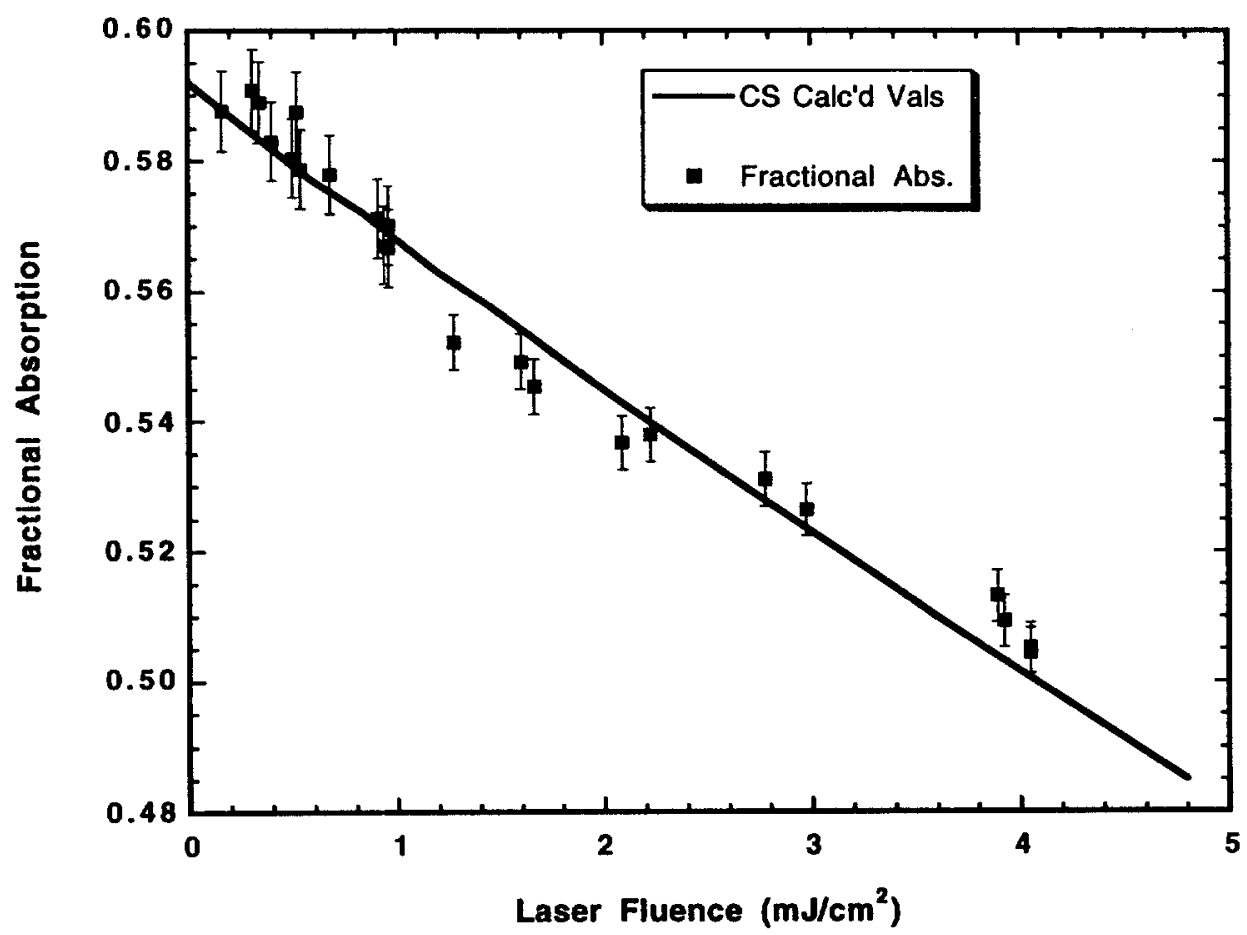

Figure 8. Experimental bleaching results for $\mathrm{HCl}$ in one atmosphere of air verses calculation

The best fit value to the data is around $k_{23}^{-1}=300$ psec which is a factor of 3 longer than assumed in Table 1 from the transition linewidth. This translates to a saturation fluence in Table 1 being too large by a factor of 3 , giving a value of $0.9 \mathrm{~mJ} / \mathrm{cm}^{2}$ rather than $2.6 \mathrm{~mJ} / \mathrm{cm}^{2}$. This then sets the saturation requirement for aperture energy per pulse at $\sim 100$ millijoules. Based upon comparative transition line strengths, HF is expected to be saturated at half this aperture energy, or 50 millijoules. These estimates are based on diffraction limited pulses and neglect beam distortion and breakup due to atmospheric effects. However, when working in the linear regime below saturation, redistribution of intensity in the beam profile will not affect the average degree of saturation. This circumstance is expected to prevail in near normal illumination geometries.

The second experimental setup is shown in Fig. 9. Here the IR beam is sent to a pellicle beamsplitter which splits off a probe pre-pulse of around $5-10 \%$ of the bleaching pulse, which goes on to a variable length, multipass cell. This delayed pulse is recombined on the pellicle and sent to the sample cell and a fast InSb detector. 


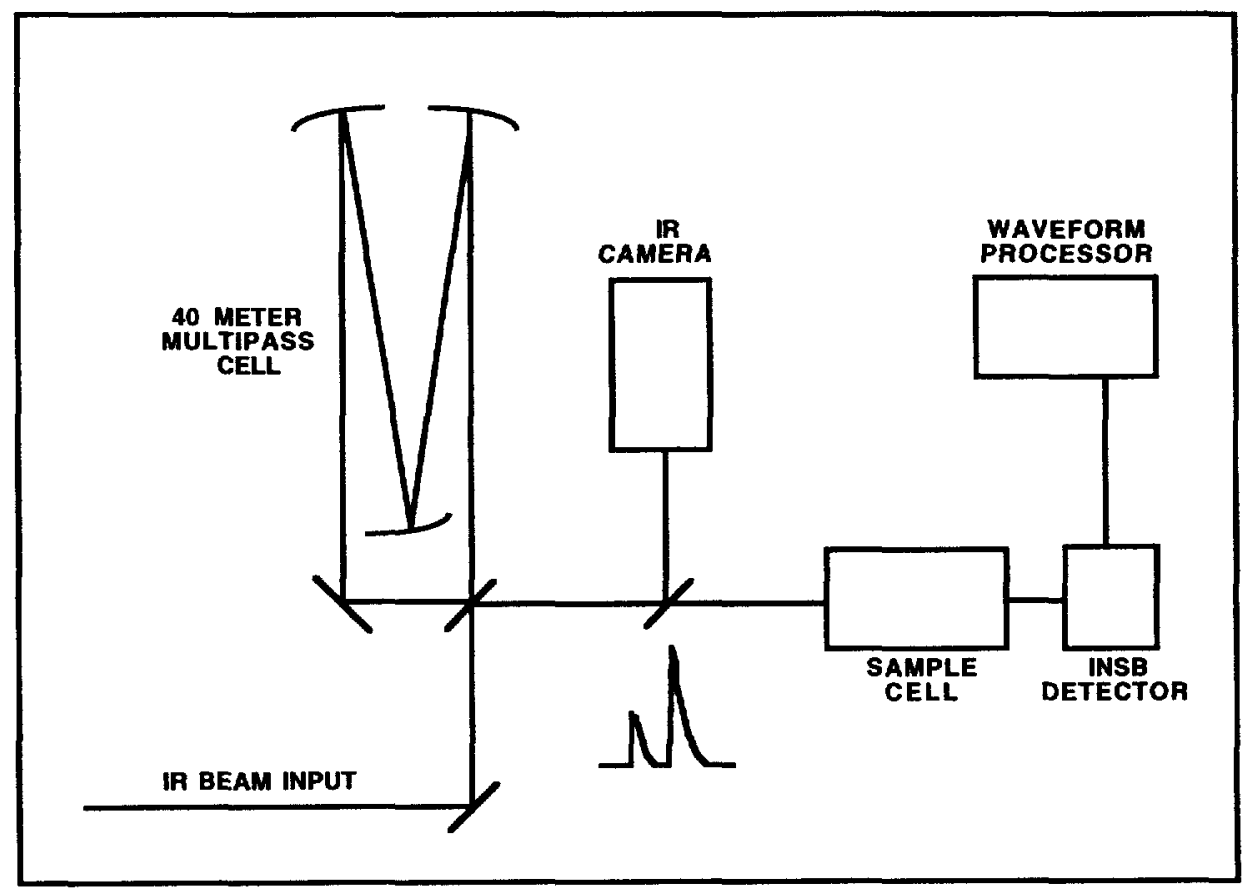

Figure 9. Optical layout for temporal two pulse experiments.

This setup was used to determine and compare measurement precision with that obtained in the two detector experiment shown in Fig. 7. The absolute precision attainable is very important because higher precision relaxes the laser fluence requirement to measure a specified absorption level. This setup was also used to determine the bleaching effect for various ratios of probe/bleach pulses, since in this setup, the weaker probe pulse also causes some bleaching to occur. Many experiments were performed to optimize all of the variables that directly affect precision such as the type of detector preamplifiers (TIA vs. voltage), data acquisition schemes, beamsplitter types and orientation ( $0^{\circ}$ vs. $90^{\circ}$ incidence), twobeam replica effects, etc. Variables which were not considered but are probably very important were laser beam quality and InSb detector element size (to eliminate possible beam overfilling). The laser beam due to the method of generation(via DFM), was of very poor quality and the area of the InSb detector used in the studies ( $0.5 \mathrm{~mm}$ diameter) was marginally larger than the focused laser spot size (which was of course larger than diffraction limited due to the poor beam quality).

The results from both sets of experiments showed similar precision. Long term drift of unknown origin was evident in attempts to average more than $\sim 500$ laser pulses at a $10 \mathrm{~Hz}$ repetition rate. Typical fractional rms noise figures for $200-500$ 
pulses was around $0.1 \%$, with $0.05 \%$ being a best case value for the single detector experiment. By further splitting the double pulse into a replicated set of two pulses, (giving a 'ratio of ratios') should allow measurements to achieve even better precision values. Bleaching ratios using the setup in Fig. 10 ranged between 4 and $8 \%$ by placing a sample cell in and out of the focal point of a $250 \mathrm{~mm}$ focal length lens. These values are lower than expected when compared with the saturation studies using the spatially separated pulses (Fig. 9) for reasons that are not completely understood. Possible explanations involve consideration of the average bandwidth mismatch between the laser and the pressure broadened sample as well as the shot-to-shot bandwidth variations. Furthermore, shot-to-shot wavelength jitter was also observed and serves to dilute the bleaching effect at absorption line center.

\section{DISCUSSION AND CONCLUSIONS}

We have demonstrated both in calculations and experiments a new laser based remote sensing system that directly addresses the major measurement difficulties of laser-detection of chemical effluents by uniquely "tagging" molecules for identification through the phenomenon of optical pumping. The technique simultaneously solves three major problems inherent with conventional DIAL measurements. Conventional DIAL relies on differential absorption between an on-resonant and an off-resonant wavelength that generates non-correlated speckle patterns which prevent a precise background determination. The conventional solution to this problem is use of brute force averaging over a large number of independent speckle lobes. Second, absorption interferences caused by other molecules, aerosols or by ground features (in the case of topological backscattering) require multiple wavelength measurements to understand the nature of the interferences fully. Both remedies require increased acquisition time, which may not be practical in potential field applications. Lastly, the OPAL technique allows precise identification of the unknown absorber with as few as two laser wavelengths, since only a fortuitous coincidence of spectroscopic constants would allow two molecules to absorb at a certain laser wavelength and be then bleached at exactly the same second wavelength. This is in contrast to conventional DIAL which only gains confidence in identification with an increasing number of laser wavelengths. However, there are circumstances where the bleached spectra may not have nicely defined bleaching partners. Large asymmetric rotor molecules could have such poorly organized energy levels that pumping a cluster of lines at one wavelength could result in a scattered structure of co-bleached lines. In some cases, the scattered bleached lines of one molecule could overlap those of another.

In the laboratory, we have demonstrated bleaching fluences of $0.9 \mathrm{~mJ} / \mathrm{cm}^{2}$ for $\mathrm{HCl}$ in one atmosphere of air, well below the eyesafe limit of $10 \mathrm{~mJ} / \mathrm{cm}^{2}$ in the midIR. Calculations show similar bleaching fluences for other molecules of increasing molecular size and complexity. Precision values of $0.05 \%$ have been demonstrated in the laboratory and paths to increase this value further have been identified. 
Let's now consider the pulse energy required for the remote detection of chemicals in a distant plume. Consider a platform located at some distance $(\mathrm{H})$ with a projection optic having a diameter $\mathrm{D}$. Using a $\mathrm{TEM}_{00}$ laser mode having a full width at half maximum (FWHM) equal to $2 \mathrm{D} / \pi$, providing $99 \%$ projection efficiency, the $86 \%$ encircled power area at the plume will be given under diffraction limited conditions as

$$
\begin{gathered}
(\text { spot area }) \times(\text { solid angle })=\lambda^{2} \\
(\text { spot area })=\pi(\lambda H / D)^{2}
\end{gathered}
$$

Operating near 3 microns in wavelength from a platform at $5 \mathrm{~km}$ with a $30 \mathrm{~cm}$ projection aperture, the spot area under these conditions will be approximately 80 $\mathrm{cm}^{2}$. At a saturation fluence of $1 \mathrm{~mJ} / \mathrm{cm}^{2}$ this sets an energy requirement of $80 \mathrm{~mJ}$ per pulse. Because of the high precision of this approach, one could operate well below the saturation fluence level and consequently require less aperture pulse energy. For example, with a target absorbance of $0.1 \%$ in the plume and a measurement precision of $0.01 \%$, then the fluence requirements can be relaxed by a factor of 10 , now operating in the 8 millijoule range for $\mathrm{HCl}$ as shown above. This level of precision is anticipated with implementation of the replicated pulse normalization scheme.

\section{ACKNOWLEDGMENT}

We acknowledge the contribution of F. Serduke at LLNL, who provided the Runge-Kutta code and who developed the rate equation code used in the model calculations. We also acknowledge the Lawrence Livermore National Laboratory LDRD Program for its support of this work. This work was performed under the auspices of the U.S. Department of Energy by the Lawrence Livermore National Laboratory under contract W-7405-Eng-48.

\section{REFERENCES}

1. see, for example, E. Zanzottera, "Differential absorption Lidar techniques in the determination of trace pollutants and physical parameters of the atmosphere", Crit. Rev. Anal Chem. 21, 279, (1990).

2. J. W. Goodman, "Statistical properties of laser speckle patterns", Laser speckle and related phenomena", J. C. Dainty, Ed., p 9, Springer-Verlag, (1975).

3. J. C. Petheram, "Differential backscatter from the atmospheric aerosol: the implications for IR differential absorption lidar", Appl. Opt. 20, 3941, (1981).

4. Molecules and Radiation, J. Steinfeld, MTT Press, (1985).

5. H. Haugen, et al., J. Chem. Phys. 80, 1839, (1984). 


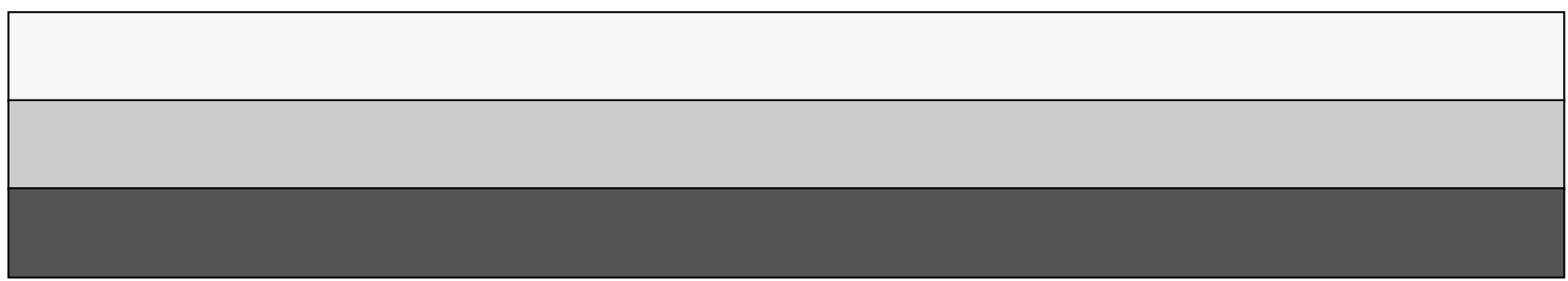

